\title{
Cell-free protein engineering of Old Yellow Enzyme 1 from Saccharomyces pastorianus.
}

Leann T. Quertinmont, Stefan Lutz*

Department of Chemistry, Emory University, 1515 Dickey Drive, Atlanta, Georgia 30084, United States

* To whom correspondence should be addressed: $\underline{\text { sal2@emory.edu }}$

(C) 2016. This manuscript version is made available under the Elsevier user license http://www.elsevier.com/open-access/userlicense/1.0/ 


\begin{abstract}
In protein engineering, cell-free transcription/translation of linear mutagenic DNA templates can tremendously accelerate and simplify the screening of enzyme variants. Using the RApid Parallel Protein EvaluatoR (RAPPER) protocol, we have evaluated the impact of amino acid substitutions and loop truncations on substrate specificity and stereoselectivity of Old Yellow Enzyme 1 from Saccharomyces pastorianus. Our study demonstrates the benefit of systematically assessing amino acid variations including substrate profiling to explore sequence-function space.
\end{abstract}

\title{
KEYWORDS
}

Old Yellow Enzyme; Flavoenzyme; In vitro transcription/translation; RAPPER; Mutagenesis 


\section{INTRODUCTION}

Enzymes are highly efficient (bio)catalysts for asymmetric synthesis of enantiomerically pure compounds, typically displaying high enantio, regio and chemo-selectivity and performing under benign environmental conditions. ${ }^{1}$ Among the variety of biocatalytic conversions, the asymmetric reduction of alkenes by members of the Old Yellow Enzyme (OYE) family is widely use to generate up to two stereogenic centers. OYEs are flavin mononucleotide (FMN)-containing NADPH-dependent oxidoreductases. Mechanistically, the reaction proceeds via a Michael-type hydride transfer step from the reduced flavin cofactor to the substrate, followed by stereospecific protonation to yield the trans-product (Fig. 1). The FMN is then reduced by NADPH, regenerating the flavin cofactor and completing the catalytic cycle.

Contributing to their popularity in biotechnology and the pharmaceutical industry, OYEs tolerate a wide range of $\alpha, \beta$-unsaturated ketones, aldehydes and carboxylic acid derivatives, as well as nitroalkenes, nitriles, and nitroaromatics as substrates. ${ }^{3-11}$ Nevertheless, the need for increased catalytic activity and stereo-selectivity of OYEs for specific substrates have inspired protein engineers to improve upon the natural diversity of these biocatalysts. A number of structure-guided site-directed and sitesaturation mutagenesis studies, as well as directed evolution experiments have yielded OYE variants with improved catalytic properties. ${ }^{12-20}$ These engineering efforts also identified several experimental challenges and limitations related to heterologous expression and test substrates and have led us to explore new strategies for evaluating libraries of OYE variants from Saccharomyces pastorianus (OYE1). ${ }^{21}$ Specifically, we switched to in vitro transcription/translation using the chemically-defined "protein synthesis using recombinant elements" (PURE) system. ${ }^{22}$ Following initial studies in PURE to eliminate cellular background reaction(s) and accelerate library screening, we continued to develop the cell-free concept to include the use of linear DNA as template for protein synthesis, enabling direct use of PCR products for functional evaluation of the corresponding native and engineered enzymes through a protocol named RAPPER (RApid Parallel Protein EvaluatoR). ${ }^{23}$ 


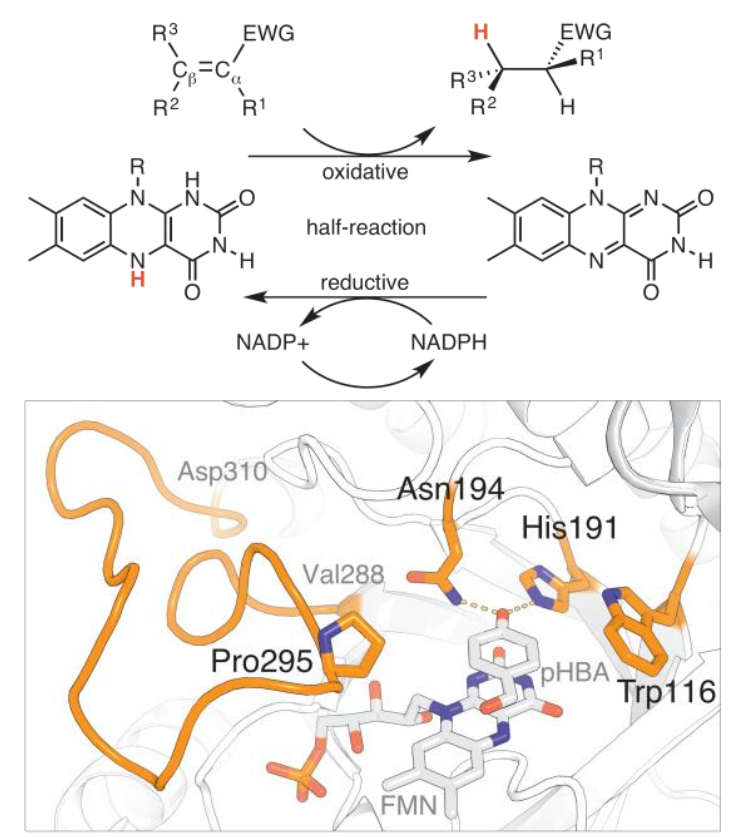

Figure 1: OYE-catalyzed trans-hydrogenation of alkenes activated by an electron-withdrawing group (EWG). The asymmetric reduction of substrate via hydride transfer (shown in red) as part of the oxidative half-reaction (in reference to flavin cofactor) is coupled with cofactor regeneration by NADPH. The crystal structure of OYE1 with substrate analog $p$-hydroxy-benzaldehyde (pHBA) bound in the active site (PDB: 1K03) ${ }^{2}$ shows the substrate's hydrogen bonding interactions with H191 and N194. The positions of $\mathrm{W} 116$ and P295, as well as the loop $\beta 6$ region (V288-D310) are highlighted.

We have now started to apply RAPPER for systematic protein engineering studies of OYE1, as well as to expand the RAPPER protocol to include multi-site mutagenesis, InDels variations, and substrate profiling. Besides validating the impact of amino acid replacements at position W116 in OYE1 on catalytic activity and stereoselectivity, we are employing RAPPER to study the functional contributions of the loop $\beta 6$ region in OYE1 by alanine-scanning and site-specific mutagenesis, as well as 
loop truncation. Finally, activity data for individual amino acid changes of two key active site residues at positions H191 and N194 were measured.

\section{RESULTS AND DISCUSSION}

A first set of systematic amino acid replacements using RAPPER focused on position W116 in OYE1. The tryptophan residue lines the active site binding pocket and substitutions at this position have been shown to be highly effective in reversing the enzyme's diastereoselectivity for reduction of $(S)$ carvone. ${ }^{13,18}$ To validate these findings in RAPPER, we employed the previously reported two-step fragment overlap extension PCR to create linear template DNA followed by in vitro transcription/translation to prepare five $\mathrm{W} 116 \mathrm{x}$ variants $(\mathrm{x}=\mathrm{A}, \mathrm{V}, \mathrm{I}, \mathrm{N}$, and $\mathrm{F}){ }^{23}$ Cell-free protein synthesis was performed at $10-\mu \mathrm{l}$ reaction scale to minimize sample evaporation and reduce variability in activity assays. However, protein expression levels were sufficiently high to split the reaction mixture after protein synthesis into $2-\mu 1$ aliquots for the subsequent assessment of catalytic activity, significantly broadening and accelerating enzyme characterization by allowing for parallel testing of up to four substrates. As summarized in Fig. 2, each W116x variant could be evaluated for reduction of (S)-carvone, methyl-2-(hydroxymethyl)acrylate, geranial, and neral in a single experiment. In comparison to wild type OYE1, the rates of conversion for selected variants showed greater than two-fold improvements for $(S)$ carvone and methyl-2-(hydroxymethyl)acrylate, respectively, while activity for geranial and neral was generally lower. Our routine analysis of assay products by chiral GC further confirmed the reversed stereoselectivity of selected W116x variants for $(S)$-carvone, consistent with previous literature reports. ${ }^{18}$ Overall, these single-time point measurements do not provide sufficient information to rationalize the underlying cause for the performance differences of our W116x variants with these substrates. Instead, they offer a rapid approach to establish substrate profiles for native and engineered enzymes, helping with identifying lead candidates for further in-depth characterization and exploring the generality of amino acid changes in engineered biocatalysts. 

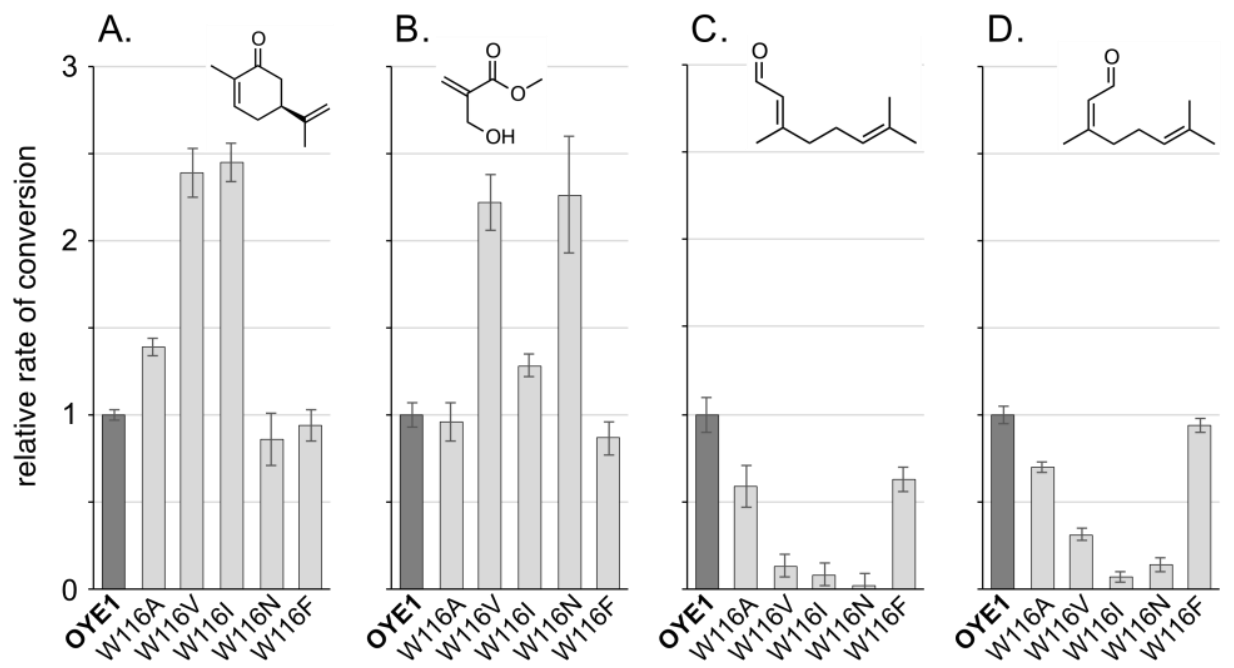

Figure 2: Rate of conversion for selected W116 variants of OYE1 relative to wild type enzyme. a.) (S)-carvone, b.) methyl-2-(hydroxymethyl)acrylate, c.) geranial, and d.) neral by RAPPER. Full experimental data including observed changes in stereoelectivity are summarized in Table S1. The results were consistent with previously reported data. ${ }^{18,25}$

A second region of interest in OYE1 is loop $\beta 6$. The extended loop region stretches from amino acid residues 288 to 310 , constituting a lid that caps the active site binding pocket (Fig. 1). Previous circular permutation studies have shown significant catalytic rate enhancements upon cleavage of peptide linkages in this loop. ${ }^{21,24}$ These functional changes were explained through changes in the loop's conformational flexibility. To further probe the role of loop $\beta 6$ on enzyme function, we conducted alanine-scanning mutagenesis of the entire region and measured its impact on $(S)$-carvone reduction. Using the RAPPER protocol enabled parallel processing of all 24 samples (wild type plus 23 variants) in triplicate. The subsequent activity assay showed variable positional effects of alanine substitutions on enzyme function (Fig. 3). By far the most significant improvement resulted from replacement of proline 
295, located at the very tip of the loop region. Besides impacting the neighboring F296 which lines the active site and undergoes a conformational change upon substrate binding ${ }^{18,24}$, the release of conformational restraints upon alanine substitution of the cyclic proline could explain the $>3$-fold activity gain. Subsequent time-course studies with purified P295A variant confirmed the observed functional gains (Fig. S1). Consistent with our interpretation of greater conformational flexibility of the entire loop $\beta 6$ region as a consequence of the P295A substitution, Tanokura and coworkers recently reported similar observations in their studies of Old Yellow Enzyme from Candida macedoniensis. ${ }^{26}$ The equivalent loop region in C. macedoniensis OYE shows significant movement upon crystallization in the presence or absence of substrate inhibitor $p$-hydroxybenzaldehyde and introduction of glycine at P295 resulted in rate increases for ketoisophorone reduction. These observations naturally raised the question whether P295A is the most beneficial amino acid change in OYE1 or whether alternative side chains could further

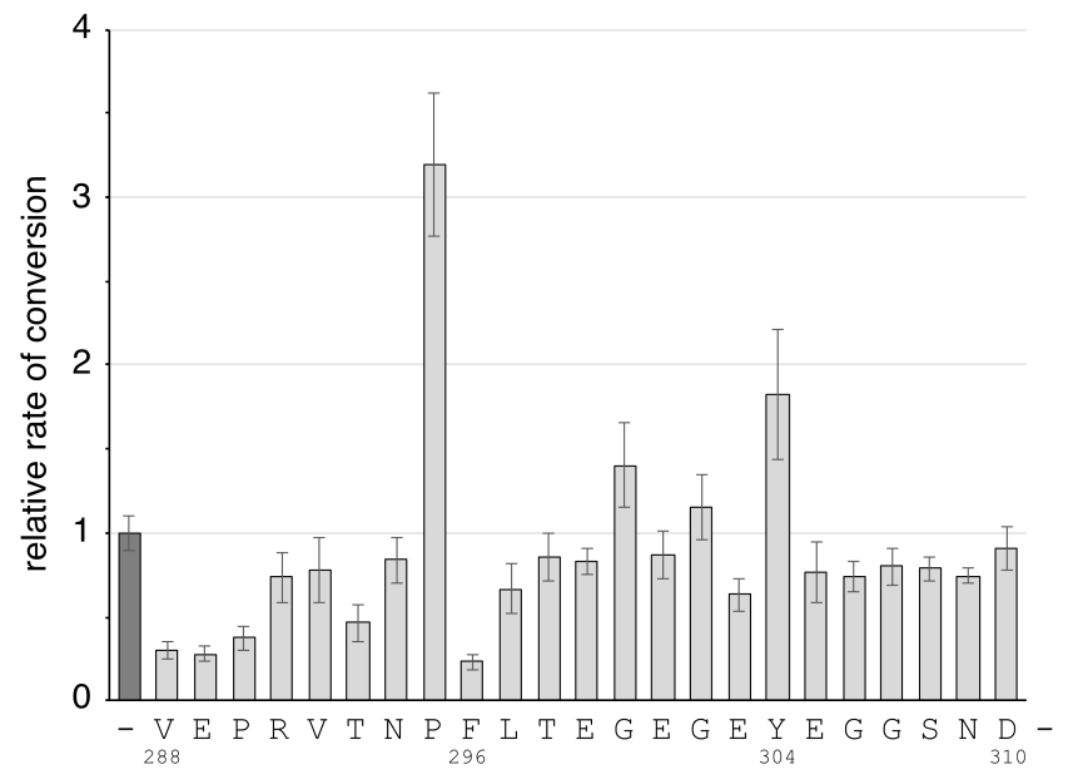

Figure 3: Relative rates of conversion for $(S)$-carvone by OYE1 variants generated via alaninescanning mutagenesis of residues in loop $\beta 6$ region (positions 288 - 310) by RAPPER. Full experimental data including observed changes in stereoelectivity are summarized in Table S2. 
modulate enzyme activity.

To further study the functional role of P295, we prepared a small, secondary library of P295x variants $(\mathrm{x}=\mathrm{G}, \mathrm{A}, \mathrm{V}, \mathrm{L}, \mathrm{M}, \mathrm{F}, \mathrm{W}$, and $\mathrm{C})$ to explore the impact of hydrophobic side chain size at this position. As for the previous W116x library, we employed RAPPER and screened all library members for activity with multiple substrates (Fig. 4). Besides (S)-carvone, methyl-2-(hydroxymethyl)acrylate, geranial, and neral, we doubled the set of test substrates by adding $(R)$-carvone, ketoisophorone, $\alpha$ methyl-trans-cinnemaldehyde and 3-methyl-2-cyclohexenone. This expanded set of substrates could again be tested in parallel by doubling the volume of cell-free protein synthesis to $20-\mu 1$ and subsequently splitting of the reaction mixture into $8 \times 2-\mu l$ activity assays. Our findings confirm the substantive
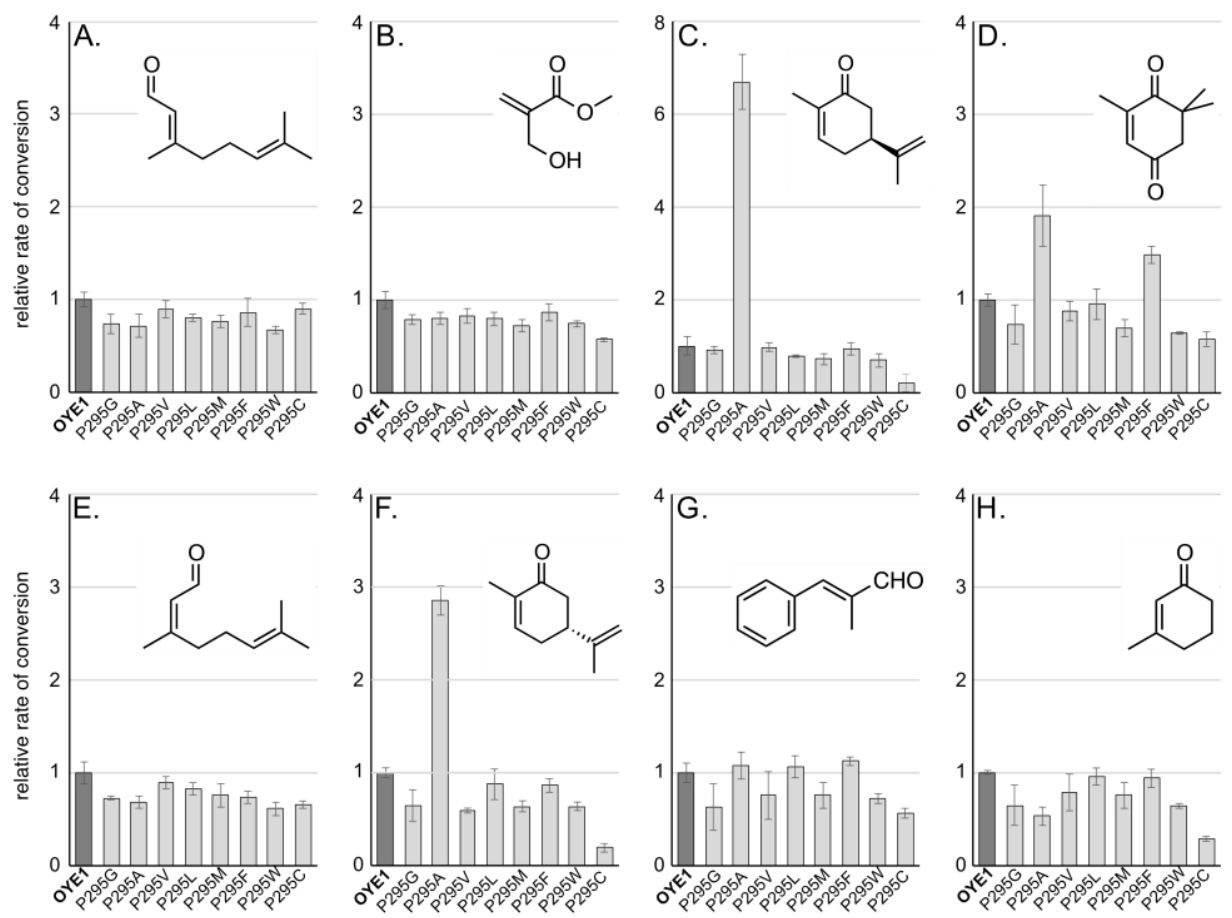

Figure 4: Substrate profiles for selected P295 variants of OYE1 by RAPPER. Relative rates of conversion were based on wild type enzyme activity for the respective substrate. a.) geranial, b.) methyl-2-(hydroxymethyl)acrylate, c.) (S)-carvone, d.) ketoisophorone, e.) neral, f.) $(R)$-carvone, g.) $\alpha$-methyl-trans-cinnemaldehyde and h.) 3-methyl-2-cyclohexenone. Full experimental data are summarized in Table S3. 
functional gains of OYE variant P295A of three and seven-fold for $(R)$ and $(S)$-carvone, respectively. Two-fold activity gains were also observed with ketoisophorone. Interestingly, the anticipated functional gains in variant P295G due to even greater conformational flexibility did not materialize. Furthermore, the functional benefits of P295A seem to only apply to a subset of substrates (Fig. 4C, D, F). For the other five test substrates (Fig. 4A, B, E, G, H), none of the substitutions in our P295x library seem to have any significant functional consequence. The distinct behavior of OYE variants with different substrates could be explained by variations in the enzymes' rate-determining step. Our results are consistent with conformational changes in loop $\beta 6$ during alkene substrate binding as the rate-limiting step for overall turnover of carvones and ketoisophorone (Fig. 4C, D, F). In contrast, changes to the loop dynamics seem irrelevant to enzyme-substrate pairs such as geranial, neral, and methyl-2-(hydroxymethyl)acrylate where other steps along the reaction coordinate are slower than the conformational changes in the loop $\beta 6$ region. While testing this hypothesis requires extensive steady-state and rapid enzyme kinetic measurements (Orru \& Lutz, unpublished results), these findings illustrate the potentially highly specific effects of individual amino acid substitutions in connection with particular substrates. Furthermore, they stress the importance of evaluating enzyme variants with multiple substrates to assess the broader impact of amino acid substitutions.

Beyond exploring the functional consequences of amino acid replacements in enzymes, the impact of amino acid insertions and deletions can offer an additional dimension in protein engineering. ${ }^{27,28}$ Such a strategy is particularly attractive for altering loop size and structure, hence we prepared a small set of OYE1 variants with deletions of up to five amino acid residues in loop $\beta 6$. Centered around position F296 at the loop tip, the RAPPER protocol was used to generate three variants; $\Delta 1$ (F296 deletion), $\Delta 3$ (F296 - T298 deletion) and $\Delta 5$ (P295 - E299 deletion) and functional changes were assayed on four substrates (Fig. 5). Our results unfortunately showed little or no significant benefit of such loop changes. The rates of reduction of the three deletion variants for $(S)$-carvone, geranial, and neral are consistently slightly lower than native OYE1 (Fig. 5A, C, D). For methyl-2(hydroxymethyl)acrylate (Fig. 5B), variant $\Delta 1$ shows a rather dramatic decline in activity, yet the removal 


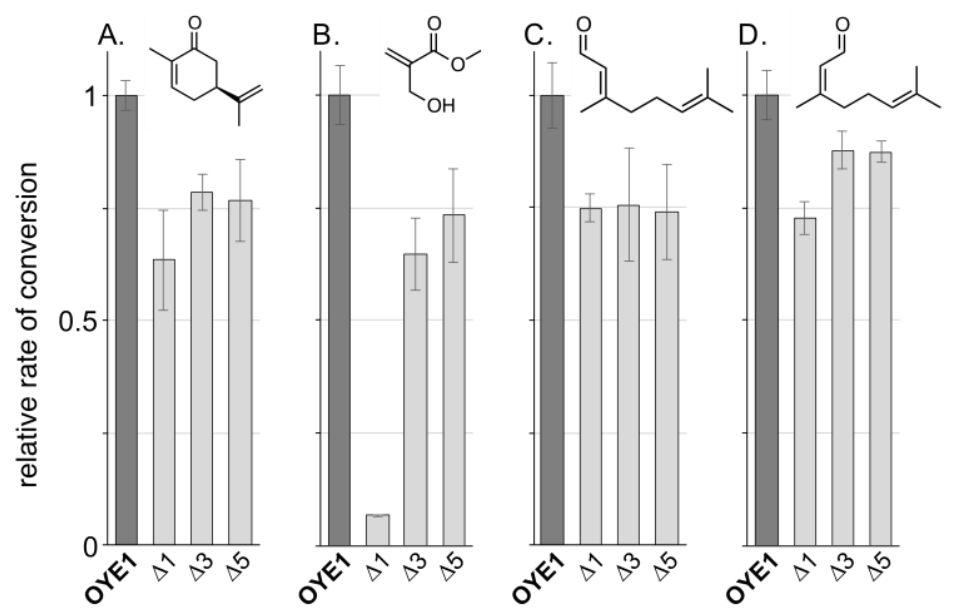

Figure 5: Impact of deletions in loop $\beta 6$ region of OYE1 on catalytic activity. Changes in relative

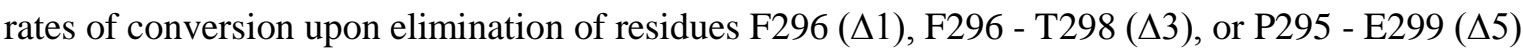
were assessed on four substrates: a.) (S)-carvone, b.) methyl-2-(hydroxymethyl)acrylate, c.) geranial, and d.) neral by RAPPER.

of additional residues in $\Delta 3$ and $\Delta 5$ largely restores catalytic activity to levels seen for the other substrates.

Finally, we applied RAPPER to explore amino acid substitution effects at positions H191 and N194, two key active site residues in OYE1. The side chains of these two amino acids typically form hydrogen bonding interactions with the substrate's electron-withdrawing group, facilitating a shift in electron density at the neighboring alkene which activates its $\beta$-position for hydride transfer from the nearby reduced flavin cofactor (Fig. 1). ${ }^{29}$ Early studies by Massey and coworkers successfully swapped amino acids at positions 191 and 194 although detrimental to catalytic activity. ${ }^{30}$ More recently, the Bommarius group reported on the ene-reductase activity (or more precisely the absence thereof) of an H191A/N194A variant of Kluyveromyces lactis OYE, a close homolog of OYE1. ${ }^{31}$ Independent of these results, we screen two single-site OYE1 libraries (H191x where $\mathrm{x}=\mathrm{A}, \mathrm{L}, \mathrm{Q}, \mathrm{S}, \mathrm{C}, \mathrm{N}$ or T; and N194x 
where $\mathrm{x}=\mathrm{A}, \mathrm{L}, \mathrm{Q}, \mathrm{S}, \mathrm{C}, \mathrm{H}$ or $\mathrm{T}$ ). The chosen amino acid substitutions enable broader sampling of size and hydrogen bonding capacity on enzyme function. The catalytic activities for these variants were assessed with $(S)$-carvone and methyl-2-(hydroxymethyl)acrylate (Fig. 6). For the reduction of $(S)$ carvone, individual amino acid replacements at H191x proved highly detrimental (Fig. 6A). Except for residual activity levels with $\mathrm{H} 191 \mathrm{~A}$, all tested substitutions at this position abolished catalytic activity. The same reaction seems tolerant of substitutions at N194 (Fig. 6B). Although activity levels dropped in
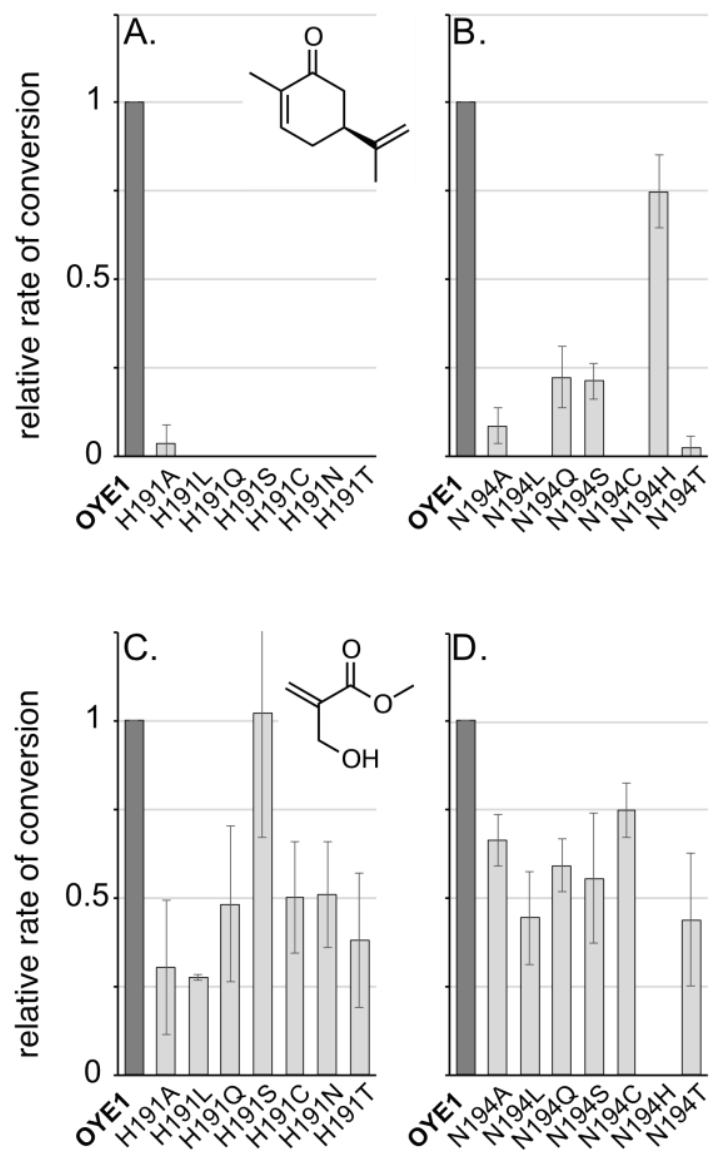

Figure 6: Effects of amino acid replacements on H191 and N194, key residues in the OYE1 active site, on catalytic activity. Relative rates of conversion were based on wild type enzyme activity for two substrates: a-b.) (S)-carvone and c-d.) methyl-2-(hydroxymethyl)acrylate by RAPPER. Full experimental data are summarized in Table S4. 
all variants, declines varied from minor (N194H) to moderate (N194S, Q) to major (N194A, T, C, L). These findings clearly suggest for H191 to play a key functional role in (S)-carvone binding and/or electronic activation and for N194 to serve in its support. Separately, we evaluated the single-variant libraries with methyl-2-(hydroxymethyl)acrylate (Fig. 6C,D) The impact of H191 and N194 variations on conversion of this second substrate differ significantly from the results obtained for $(S)$-carvone. All but one member of the two libraries $(\mathrm{N} 194 \mathrm{H})$ retain conversion rates of $>25 \%$ relative to OYE1, suggesting that neither of these positions plays the same critical functional role. More importantly, our results demonstrate the highly substrate-dependent effects of amino acid changes in OYE1. More detailed experimental studies with these alanine variants to explore the structural and functional consequences of these substitutions is in progress.

\section{CONCLUSION}

Protein engineering of oxidoreductases in the Old Yellow Enzyme family continue to be of great interest for developing novel biocatalysts. Our present study used small, focused libraries to systematically probe the functional consequences of specific residues and regions in OYE1. In contrast to similar previous studies, the application of RAPPER enables faster and more efficient, semi-quantitative evaluation of enzyme variants. Beyond changes to individual amino acid positions, fragment overlap extension PCR has successfully been applied to generate gene template for exploring functional consequences of residual InDels (insertions/deletions). Furthermore, RAPPER's capacity to support parallel screening of engineered biocatalysts with multiple substrates enables a more comprehensive assessment of impact and contributions of individual amino acid substitutions. Our present results for OYE1 highlights the potential benefits of substrate profiling in protein engineering studies, averting the risk of misleading results from usage of a model substrate. Functional gains of amino acid replacements such as P295A or changes in stereoselectivity observed for W116I show significant substrate dependence. Similar to other high-throughput screening platforms, RAPPER does have limitations due to the small 
sample sizes. Protein yields for enzyme variants from 10- $\mu$ l PURE reactions are to low to assess questions of expression level, foldability and stability of individual candidates in detail. Furthermore, amino acid changes may result in altered inhibitory properties and substrate binding affinity which can affect the outcome of the screening assay and complicate direct comparison of variants. Lead candidates are therefore routinely evaluated in follow-up studies using traditional biochemical and biophysical methods and these results have validated the semi-quantitative nature of RAPPER data, making the protocol useful for initial screening of enzyme libraries. In summary, the use of cell-free systems offers a simple, versatile and very effective tool to accelerate the discovery process for tailored biocatalysts.

\section{EXPERIMENTAL}

4.1 General information. The PURExpress ${ }^{\circledR}$ kit was purchased from New England Biolabs (Ipswitch, MA). All primers used were synthesized by Integrated DNA Technologies (Coralville, IA). All other reagents were purchased from Sigma Aldrich (St. Louis, MO) unless otherwise specified. GC spectra were obtained on an Agilent Technologies 6850 GC instrument equipped with a chiral CycloSil-B column ( $30 \mathrm{~m} \times 0,32 \mathrm{~mm} / 0.25 \mu \mathrm{m}$, Agilent, Santa Clara, CA) using hydrogen as a carrier gas (flow rate 1.8 $\mathrm{mL} / \mathrm{min}$ ) and an FID detector (detector temperature $200^{\circ} \mathrm{C}$, split ratio $25: 1$ ).

4.2 Creating linear templates of OYE variants. All mutations were prepared by a two-step fragment overlap extension PCR protocol using pET14b-OYE1 as a template. ${ }^{21,23}$ Mutagenic primer pairs are listed in Table S1 and were used in combination with universal flanking primers carrying a 5'-desthiobiotin-tag for gel-free fragment purification (forward: ${ }^{5}$-desthiobiotin-CTCGATCCCGCGAAA TTAATACGACT3'; reverse: ${ }^{5}$-desthiobiotin-CAGCAAAAAACCCCTCAAGACCCG- ${ }^{3}$ ). Following the first PCR, reaction mixtures were diluted with an equal volume of distilled water and added to high-capacity streptavidin agarose beads (Pierce, Grand Island, NY) pre-equilibrated in binding buffer ( $5 \mathrm{mM}$ Tris- $\mathrm{HCl}$ 
(pH 7.5), $0.5 \mathrm{mM}$ EDTA, $1 \mathrm{mM} \mathrm{NaCl}$ ). After incubation at $21{ }^{\circ} \mathrm{C}$ for $15 \mathrm{~min}$, the mixture was centrifuged at 13,000 rpm for $3 \mathrm{~min}$ and the supernatant discarded. The streptavidin-bound fragments were washed three times with $100 \mu \mathrm{L}$ binding buffer, followed by incubation in $50 \mu \mathrm{L}$ elution buffer ( $4 \mathrm{mM}$ biotin, 10 $\mathrm{mM}$ Tris- $\mathrm{HCl}, \mathrm{pH} 8.5$ ) at $21{ }^{\circ} \mathrm{C}$ for $10 \mathrm{~min}$. The mixture was centrifuged at $13,000 \mathrm{rpm}$ for $3 \mathrm{~min}$ and the clear supernatant containing fragment DNA was collected. For the second PCR to reassemble fragments into the full-length genes, aliquots of the fragment DNA were mixed with universal flanking primers (same sequence as for the first PCR but without 5'-tags). Full-length PCR products were purified using the QIAgenPCR Purification kits (Qiagen, Valencia, CA) and quantified by UV absorbance spectroscopy at $280 \mathrm{~nm}$.

4.3 In vitro transcription/translation of linear DNA. Linear DNA (PCR products) were used as templates for protein expression of selected OYE variants to access their catalytic activities as described previously. ${ }^{23}$ Briefly, protein synthesis was performed by in vitro transcription/translation (IVTT), using the PURExpress system and reaction products were directly tested for ene-reductase activity with selected substrates of interest. With each set of experiments, wild type OYE1 was run as a positive control while dihydrofolate reductase (provided by NEB) served as negative control. Typically, protein synthesis was terminated after $2.5 \mathrm{~h}$ incubation at $37^{\circ} \mathrm{C}$ by storing the mixture on ice.

4.4 Large-scale protein expression and purification. To obtain larger quantities of purified OYE variants, PCR products were cloned into pET-14b (Novagen) via NcoI/XhoI restriction sites and the plasmid DNA transformed into E. coli BL21(DE3) pLysS cells. Individual colonies were grown overnight in $5 \mathrm{~mL}$ of $2 \mathrm{YT}$ media containing ampicillin $(100 \mu \mathrm{g} / \mathrm{mL})$ at $37{ }^{\circ} \mathrm{C}$. An aliquot of the overnight culture was then used to inoculate $250 \mathrm{~mL}$ of $2 \mathrm{YT}$ containing ampicillin $(100 \mu \mathrm{g} / \mathrm{mL})$. Following incubation at $37{ }^{\circ} \mathrm{C}$ until the culture reached an $\mathrm{OD}(600)$ of 0.7 , heterologous protein expression was induced by addition of IPTG (final concentration: $0.4 \mathrm{mM}$ ) for $18 \mathrm{~h}$ at $20{ }^{\circ} \mathrm{C}$. Cells were pelleted by centrifugation at $4{ }^{\circ} \mathrm{C}, 4000 \mathrm{~g}$ for $20 \mathrm{~min}$, the clear supernatant removed and pellets stored at $-20{ }^{\circ} \mathrm{C}$ until further usage. 
For protein purification, a cell pellet was resuspended in $24 \mathrm{~mL}$ of buffer $\mathrm{A}$ (40 mM Tris- $\mathrm{HCl}$ (pH 8.0), $20 \mathrm{mM} \mathrm{NaCl}$ ) and mixed with $200 \mu \mathrm{L}$ protease inhibitor cocktail (Sigma) and $20 \mu \mathrm{L}$ of benzonase (Novagen). After $30 \mathrm{~min}$ storage on ice, cells were lysed by sonication. The suspension was centrifuged at $4{ }^{\circ} \mathrm{C}, 10,000 \mathrm{~g}$ for $30 \mathrm{~min}$ and the clear supernatant was loaded on a HiTrap Q FF (5 mL) anion-exchange column, pre-equilibrated in buffer A. The resin was washed with 2 column volumes (CVs) buffer A, followed by a linear gradient over $10 \mathrm{CVs}$ to $100 \%$ buffer B (40 mM Tris- $\mathrm{HCl}$ (pH 8.0), $1 \mathrm{M} \mathrm{NaCl})$. Protein elusion was monitored by $\mathrm{UV}$ absorption $(280 / 460 \mathrm{~nm})$ and product fractions were collected, followed by concentration and buffer-exchange into buffer $\mathrm{C}(40 \mathrm{mM}$ Tris- $\mathrm{HCl}(\mathrm{pH} 8.0), 300$ $\mathrm{mM} \mathrm{NaCl}$ ) using a Millipore filter unit (MWCO: $10 \mathrm{kDa}$ ). The protein sample was further purified via size exclusion chromatography (Superdex 200, 10/300 GL column) in buffer C. Product fractions were pooled, concentrated as described above, and analyzed by SDS-PAGE.

4.5 Enzymatic activity assay for OYE1 variants. Ene-reductase activity assays were performed at ambient temperature under anaerobic conditions (Coy Laboratory, Grass Lake, MI). For preparing reaction stock solution, individual substrates (see below) were dissolved in $50 \mathrm{mM}$ Tris- $\mathrm{HCl}(\mathrm{pH} 7.5$ ), supplemented with $200 \mu \mathrm{M} \mathrm{NADP}{ }^{+}, 100 \mathrm{mM}$ glucose, and glucose dehydrogenase (GDH) from Thermoplasma acidophilum ( 2 units for IVTT reactions, 5 units for purified enzyme reactions). Individual substrate concentrations were chosen to ensure $\mathrm{v}_{\max }$ conditions or maximum solubility. At these substrate levels, reaction times were adjusted for $10-50 \%$ substrate conversion.

To assay OYE activity in IVTT experiments, $20 \mu \mathrm{L}$ of the reaction stock solution was mixed with 2 to $10 \mu \mathrm{L}$ IVTT reaction mixture and the total assay volume was adjusted to $30 \mu 1$ with $50 \mathrm{mM}$ Tris- $\mathrm{HCl}$ (pH 7.5). To assays purified OYE1 variants, the enzyme (final concentration: $250 \mathrm{nM}$ ) was added to 500 $\mu 1$ reaction stock solution. Reaction progress was monitored by removing $30-\mu l$ aliquots from the assay solution and quenching them by mixing thoroughly with $30 \mu \mathrm{L}$ of ethyl acetate containing $1 \mathrm{mM}$ cyclohexanone as internal standard. A sample of the organic phase was collected and analyzed by GC (protocols: see below). The enantio/diasteriomeric excess were calculated by integration of product and 
substrate peak areas. Relative rates of conversion for individual substrates were calculated by dividing the measured rate of conversion for OYE1 variant over the corresponding rate for wild type OYE1.

4.5.1 (S)-Carvone. Reaction time: $4 \mathrm{~h}$; Final substrate concentration: $10 \mathrm{mM}$. GC protocol: $90{ }^{\circ} \mathrm{C}$, hold 5 $\min$, then $1{ }^{\circ} \mathrm{C} / \mathrm{min}$ to $120{ }^{\circ} \mathrm{C}$ (retention times for $(S)$-carvone $=27.1 \mathrm{~min},(1 R, 4 S)$-dihydrocarvone $=22.9$ $\min ,(1 S, 4 S)$-dihydrocarvone $=22.6 \mathrm{~min})$.

4.5.2 (R)-Carvone. Reaction time: $4 \mathrm{~h}$; Final substrate concentration: $2 \mathrm{mM}$. GC protocol: $90{ }^{\circ} \mathrm{C}$, hold 5 $\min$, then $1{ }^{\circ} \mathrm{C} / \mathrm{min}$ to $120{ }^{\circ} \mathrm{C}$ (retention times for $(R)$-carvone $=27.2 \mathrm{~min},(1 R, 4 R)$-dihydrocarvone $=22.3$ $\min ,(1 S, 4 R)$-dihydrocarvone $=22.5 \mathrm{~min})$.

4.5.3 Neral. Reaction time: 6 h; Final substrate concentration: $1 \mathrm{mM}$. GC protocol: $80{ }^{\circ} \mathrm{C}$ ramped at 2.5 ${ }^{\circ} \mathrm{C} / \mathrm{min}$ to $115{ }^{\circ} \mathrm{C}$, hold for $3 \mathrm{~min}$ (retention times for neral $=13.0 \mathrm{~min}$, citronellal $=8.9 \mathrm{~min}$ ).

4.5.4 Geranial. Reaction time: $0.5 \mathrm{~h}$; Final substrate concentration: $5 \mathrm{mM}$. GC protocol: $80{ }^{\circ} \mathrm{C}$ ramped at $2.5^{\circ} \mathrm{C} / \mathrm{min}$ to $115^{\circ} \mathrm{C}$, hold for $3 \mathrm{~min}$ (retention times for geranial $=14.8 \mathrm{~min}$, citronellal $=8.9 \mathrm{~min}$ ).

4.5.5 Methyl-2-(hydroxymethyl)acrylate. Reaction time: 24 h; Final substrate concentration: 5 mM. GC protocol: $60{ }^{\circ} \mathrm{C}$, hold for $1 \mathrm{~min}$, then $1{ }^{\circ} \mathrm{C} / \mathrm{min}$ to $75^{\circ} \mathrm{C}$, hold for 4 min (retention times for methyl-2hydroxymethyl acrylate $=16.6 \mathrm{~min},(R)$-3-hydroxy-2-methylpropanoate $=15.0 \mathrm{~min})$.

4.5.6 Ketoisophorone. Reaction time: 2.5 h; Final substrate concentration: $200 \mu \mathrm{M}$. GC protocol: $150{ }^{\circ} \mathrm{C}$, hold for $5 \mathrm{~min}$ (retention times for ketoisophorone $=3.26 \mathrm{~min},(R)$-levodione $=3.59 \mathrm{~min},(S)$-levodione $=$ $4.4 \mathrm{~min})$. 
4.5.7 3-Methyl-2-cyclohexenone. Reaction time: 24 h; Final substrate concentration: $1 \mathrm{mM}$. GC protocol: $150{ }^{\circ} \mathrm{C}$, hold for $5 \mathrm{~min}$ (retention times for 3-methyl-2-cyclohexenone $=2.76 \mathrm{~min}$, 3-(S)methylcyclohexanone $=2.06 \mathrm{~min})$.

4.5.8 $\alpha$-Methyl-trans-cinnamaldehyde. Reaction time: $0.5 \mathrm{~h}$; Final substrate concentration: $1 \mathrm{mM}$. GC protocol: $150{ }^{\circ} \mathrm{C}$ hold for $10 \mathrm{~min}$ (retention times for $\alpha$-methyl-trans-cinnamaldehyde $=6.28 \mathrm{~min}, 2$ methyl-3-phenylpropanal $=3.82 \mathrm{~min})$.

\section{ACKNOWLEDGEMENTS}

We thank the members of the Lutz lab for helpful comments and suggestions on the manuscript. Financial support in part by the US National Science Foundation (CBET-1159434) is gratefully acknowledged.

\section{REFERENCES AND NOTES}

(1) Bornscheuer, U. T.; Huisman, G. W.; Kazlauskas, R. J.; Lutz, S.; Moore, J. C.; Robins, K. Nature 2012, 485, 185-194.

(2) Brown, B. J.; Hyun, J. W.; Duvvuri, S.; Karplus, P. A.; Massey, V. J. Biol. Chem. 2002, 277, 2138-2145.

(3) Turrini, N. G.; Hall, M.; Faber, K. Adv. Synth. Catal. 2015, 357, 1861-1871.

(4) Amato, E. D.; Stewart, J. D. Biotechnol. Adv. 2015, 33, 624-631.

(5) Brenna, E.; Crotti, M.; Gatti, F. G.; Monti, D.; Parmeggiani, F.; Powell, R. W.; Santagelo, S.; Stewart, J. D. Adv. Synth. Catal. 2015, 357, 1849-1860.

(6) Zhou, X.; Chow, H. L.; Wu, J. C. Biocatal. Biotransform. 2014, 32, 267-275. 
(7) Toogood, H. S.; Fryszkowska, A.; Hare, V.; Fisher, K.; Roujeinikova, A.; Leys, D.; Gardiner, J. M.; Stephens, G. M.; Scrutton, N. S. Adv. Synth. Catal. 2008, 350, 2789-2803.

(8) Adalbjornsson, B. V.; Toogood, H. S.; Fryszkowska, A.; Pudney, C. R.; Jowitt, T. A.; Leys, D.; Scrutton, N. S. Chembiochem 2010, 11, 197-207.

(9) Toogood, H. S.; Gardiner, J. M.; Scrutton, N. S. Chemcatchem 2010, 2, 892-914.

(10) Durchschein, K.; Hall, M.; Faber, K. Green Chem. 2013, 15, 1764-1772.

(11) Toogood, H. S.; Scrutton, N. S. Curr. Opin. Chem. Biol. 2014, 19, 107-115.

(12) Bougioukou, D. J.; Kille, S.; Taglieber, A.; Reetz, M. T. Adv. Synth. Catal. 2009, 351, 3287-3305.

(13) Padhi, S. K.; Bougioukou, D. J.; Stewart, J. D. J. Am. Chem. Soc. 2009, 131, 3271-3280.

(14) Hulley, M. E.; Toogood, H. S.; Fryszkowska, A.; Mansell, D.; Stephens, G. M.; Gardiner, J. M.; Scrutton, N. S. Chembiochem 2010, 11, 2433-2447.

(15) Hall, M.; Bommarius, A. S. Chem. Rev. 2011, 111, 4088-4110.

(16) Toogood, H. S.; Fryszkowska, A.; Hulley, M.; Sakuma, M.; Mansell, D.; Stephens, G. M.; Gardiner, J. M.; Scrutton, N. S. Chembiochem 2011, 12, 738-749.

(17) Reich, S.; Hoeffken, H. W.; Rosche, B.; Nestl, B. M.; Hauer, B. Chembiochem 2012, 13, 2400-2407.

(18) Pompeu, Y. A.; Sullivan, B.; Stewart, J. D. ACS Catal. 2013, 3, 2376-2390.

(19) Reich, S.; Kress, N.; Nestl, B. M.; Hauer, B. J. Struct. Biol. 2014, 185, 228-233.

(20) Walton, A. Z.; Sullivan, B.; Patterson-Orazem, A. C.; Stewart, J. D. ACS Catal. 2014, 4, $2307-2318$.

(21) Daugherty, A. B.; Govindarajan, S.; Lutz, S. J. Am. Chem. Soc. 2013, 135, 14425-14432.

(22) Shimizu, Y.; Inoue, A.; Tomari, Y.; Suzuki, T.; Yokogawa, T.; Nishikawa, K.; Ueda, T. Nat. Biotechn. 2001, 19, 751-755.

(23) Quertinmont, L. T.; Orru, R.; Lutz, S. Chem. Commun. (Camb.) 2015, 51, 122-124.

(24) Daugherty, A. B.; Horton, J. R.; Cheng, X.; Lutz, S. ACS Catal. 2015, 5, 892-899.

(25) Walton, A. Z.; Conerly, W. C.; Pompeu, Y.; Sullivan, B.; Stewart, J. D. ACS Catal. 2011, 1, 
989-993.

(26) Horita, S.; Kataoka, M.; Kitamura, N.; Nakagawa, T.; Miyakawa, T.; Ohtsuka, J.; Nagata, K.; Shimizu, S.; Tanokura, M. Chembiochem 2015, 16, 440-445.

(27) Rockah-Shmuel, L.; Toth-Petroczy, A.; Sela, A.; Wurtzel, O.; Sorek, R.; Tawfik, D. S. PLoS Genet. 2013, 9, e1003882.

(28) Arpino, J. A.; Reddington, S. C.; Halliwell, L. M.; Rizkallah, P. J.; Jones, D. D. Structure 2014, 22, 889-898.

(29) Fox, K. M.; Karplus, P. A. Structure 1994, 2, 1089-1105.

(30) Brown, B. J.; Deng, Z.; Karplus, P. A.; Massey, V. J. Biol. Chem. 1998, 273, 32753-32762.

(31) Park, J. T.; Gomez Ramos, L. M.; Bommarius, A. S. Chembiochem 2015, 16, 811-818. 